\title{
Beobachtungen an CdS-Kristallen nach Untersuchungen im Mikroskop und Leitfähigkeitsmessungen
}

\author{
Von L. Herforth und J. Krumbiegel
}

Aus dem Institut für Medizin und Biologie (Abteilung Biophysik), Berlin-Buch, der Deutschen Akademie der Wissenschaften zu Berlin, Direktor: Professor Dr. W. Friedrich

(Z. Naturforschg. 9a, 432-434 [1954]; eingegangen am 26. Februar 1954)

\begin{abstract}
Nach genauer Beobachtung der bei zwei verschiedenen Züchtungsarten entstehenden Kristalltypen wurde festgestellt, daß bei Umsublimation um etwa $50 \%$ mehr Einkristalle wachsen als bei Züchtung im direkten $\mathrm{H}_{2}$ S-Strom. Beobachtungen im Mikroskop ergaben, daß bei allen Kristallen mit Riefeln die Längsrichtung dieser Riefeln identisch mit einer

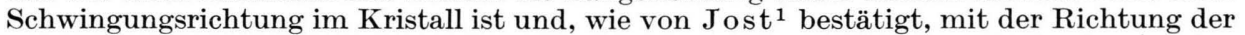
kristallographischen $c$-Achse zusammenfällt. Einkristalle zeigen im Hochvakuum eine um $42 \%$ größere mittlere Leitfähigkeit als Zwillingskristalle. Homogene Einkristalle leiten am besten. Kristalle vom Typ 2 zeigten eine um $25 \%$ größere Leitfähigkeit in Richtung der $c$-Achse als senkrecht dazu. Leitfähigkeitsuntersuchungen an Luft ergaben eine erhöhte Leitfähigkeit, wobei bei allen Kristallen ein Gleichrichtereffekt beobachtet werden konnte. Es wird versucht, diesen durch polarorientierte Adsorption von Wassermolekülen infolge der polaren $c$-Achse zu erklären.
\end{abstract}

\section{Ziel der Untersuchungen}

$\mathrm{W}$ ie an anderer Stelle ${ }^{2}$ mitgeteilt, unterscheiden wir die bei der Züchtung nach Frerichs ${ }^{3}$ im $\mathrm{H}_{2} \mathrm{~S}$-Strom entstehenden CdS-Kristalle nach fünf Typen. Gewisse Anhaltspunkte lagen nach Untersuchung einer größeren Anzahl von Kristallen der verschiedenen Typen vor, daß die mittlere lichtelektrische Leitfähigkeit der einzelnen Kristalltypen verschieden ist. Dies veranlaßte uns dazu, die Entstehung der einzelnen Typen bei der Züchtung näher zu betrachten, um dann weiterhin nach mikroskopischen Untersuchungen und Röntgenstrukturaufnahmen festzustellen, inwieweit die lichtelektrische Leitfähigkeit strukturell bedingt ist. Schließlich sollte unsere Beobachtung, daß Kristalle aller fünf Typen in Luft und im Vakuum eine bevorzugte Leitfähigkeitsrichtung aufweisen, einer genauen Prüfung im Hochvakuum unterzogen werden.

\section{Zum Wachstum der Kristalle}

Bei genauer Kontrolle der bei jeder Züchtung entstehenden CdS-Kristalle zeigte sich, daß Zwillingskristalle vorwiegend dann wachsen, wenn der $\mathrm{H}_{2} \mathrm{~S}$-Gasstrom direkt an das verdampfende Cadmium geleitet wird. Nur in Bodennähe des Schiffchens, in welchem sich das Cadmium befindet, wach-

${ }^{1}$ K. Jost, Z. Naturforschg. 9a, 435 [1954]; nachstehend.

${ }^{2}$ L. Herforth u. J. Krumbiegel, Naturwiss. 40, 270 [1952].

${ }^{3}$ R. Frerichs, Phys. Rev. 72, 594 [1947]. sen vereinzelt homogene oder geriefelte Einkristalle. Bei ungleichmäßiger $\mathrm{H}_{2} \mathrm{~S}$-Zufuhr ist somit die Möglichkeit, glasklare Einkristalle zu erhalten, wie sie allgemein bevorzugt verwendet werden, sehr gering. Diese Einkristalle erhält man in größerer Anzahl nach Umsublimation der im direkten Gasstrom entstehenden Kristalle bei etwa $1050^{\circ} \mathrm{C}$ in einer $\mathrm{H}_{2^{-}}$ Atmosphäre. In Abb. 1 sind die fünf Typen ${ }^{4}$ der Kristalle skizziert in Übereinstimmung mit der in unserer vorhergehenden Arbeit ${ }^{5}$ getroffenen Einteilung.
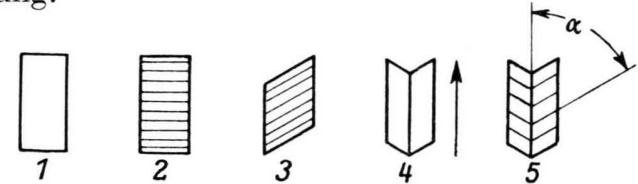

Abb. 1. Typen $1-5$ schematisch.

\begin{tabular}{|l|c|c|c|c|c|}
\hline \multirow{2}{*}{ Züchtungsart } & \multicolumn{5}{|c|}{$\begin{array}{c}\text { Anzahl der erhaltenen. Kristalle } \\
\text { nach je sieben Züchtungen }\end{array}$} \\
\cline { 2 - 6 } & Typ: 1 & 2 & 3 & 4 & 5 \\
\hline Umsublimation & 93 & 171 & 21 & 12 & 33 \\
\hline $\begin{array}{l}\text { Im direkten } \\
\text { Gasstrom }\end{array}$ & 17 & 13 & 67 & 22 & 187 \\
\hline
\end{tabular}

Tab. 1. Häufigkeitsverteilung der CdS-Kristalle über die fünf Typen bei zwei Züchtungsarten von je sieben Züchtungen.

${ }^{4}$ In dieser Arbeit ${ }^{2}$ befinden sich Photographien der einzelnen Typen.

5 Mitunter erscheinen noch einzelne wenige Zwillingskristalle, bei welchen die beiden Zwillingshälften deutlich gegeneinander geneigt sind. Diese blieben bei unseren bisherigen Untersuchungen unberücksichtigt. 
In Tab. 1 sind die Anzahlen der erhaltenen Kristalle der einzelnen Typen nach den beiden Züchtungsmethoden angegeben.

$\mathrm{Zu}$ erwähnen bleibt noch, daß alle Zwillingskristalle so wachsen, daß der von den Riefeln eingeschlossene Winkel (Abb. 1), dem wir nach unserer ersten Mitteilung Werte um $30^{\circ}, 45^{\circ}$ und $60^{\circ}$ zuordneten, in Wachstumsrichtung offen ist. Der in Abb. 1 an Typ 4 angebrachte Pfeil gibt die Wachstumsrichtung an.

\section{Mikroskopische Beobachtungen}

Die Zuordnung der Kristalle zu den fünf verschiedenen Typen erfolgt am besten nach Betrachtung im Mikroskop. Wie bereits mitgeteilt, erkennt man im Polarisationsmikroskop, daß die Längsrichtung der Riefeln identisch ist mit einer Auslöschungsrichtung bei Verwendung gekreuzter Nicols.

Frerichs ${ }^{6}$ stellte fest, daß Nadeln von sechseckigem Querschnitt mit den Seitenflächen zu geriefelten Bändern zusammenwachsen. Hiernach sollte die $c$-Achse des hexagonalen Gitters der CdSKristalle in Richtung der Riefeln fallen, was Röntgenstrukturaufnahmen von J ost bestätigten. Diese kann daher bei den geriefelten Kristallen leicht aufgefunden werden, was besonders die Justierung der Kristalle für Röntgenstrukturaufnahmen erleichtert. - An homogenen Kristallen, bei welchen die Richtung der $c$-Achsen nicht am Verlauf der Riefeln zu erkennen ist, kommt für den Verlauf der $c$-Achse eine dieser beiden Auslöschungsrichtungen in Frage. Auch hiernach ist das Auffinden der $c$-Achse in homogenen Kristallen wesentlich vereinfacht. -

Abb. $2 *$ zeigt einige Aufnahmen von Kristallen im Polarisationsmikroskop. Leider konnten hier keine Farbaufnahmen zum Abdruck kommen. Es sei aber nochmals auf die Farbenerscheinungen im polarisierten Licht hingewiesen, die bei CdS-Kristallen wegen der Eigenfarbe und der anomalen Dispersion besonders eindrucksvoll sind.

\section{Leitfähigkeitsmessungen}

Die zur Messung verwendeten Kristalle wurden keinem besonderen Aktivierungsprozeß unterzogen, so daß diese nur unbekannte Aktivatoratome enthielten.

Wir haben zu den in unserer letzten Arbeit untersuchten 80 Kristallen noch an 60 weiteren der fünf

${ }^{6}$ R. Frerichs, Naturwiss. 33, 281 [1946]. verschiedenen Typen Leitfähigkeitsmessungen vorgenommen. Die untersuchten Kristalle wurden wieder durch Bedampfen mit zwei Silberelektroden im Abstand von $1 \mathrm{~mm}$ versehen, auf Plexiglas aufgekittet und zur Messung in einen Rezipienten eingesetzt. Als Lichtquelle diente eine 30 Watt starke Scheinwerferlampe, deren weißes Licht durch eine 10-proz. $\mathrm{CuSO}_{4}$-Lösung gefiltert und mittels einer Sammellinse auf den Kristallspalt abgebildet wurde. Die an die Elektroden jeweils gelegte Saugspannung betrug 12 Volt und der zu messende Photostrom wurde durch ein Mikroamperemeter angezeigt.

Um die Aussage, da $\beta$ die einzelnen Typen von Kristallen ein unterschiedliches Verhalten in ihrem Leitvermögen aufweisen, zu erhärten, wurde die Leitfähigkeit der einzelnen Kristalle im Hochvakuum $\left(10^{-5} \mathrm{~mm} \mathrm{Hg}\right)$ bestimmt und die mittlere Leitfähigkeit (ausgedrückt in $\mu \mathrm{A} /$ Fläche oder auch $\mu \mathrm{A} /$ Volumen) berechnet. In Tab. 2 sind die Mittelwerte der errechneten Ströme an je 15 Kristallen der einzelnen Typen aufgeführt, wobei Typ 4 und 5 zusammengefaßt sind. Vergleicht man die mittlere Leitfähigkeit der Einkristalle vom Typ $1+2+3$ mit der der Zwillinge vom Typ $4+5$, so zeigen die Einkristalle eine um $42 \%$ größere mittlere Leitfähigkeit.

\begin{tabular}{|c|rrrrrr|}
\hline $\begin{array}{c}\text { Mittlere } \\
\text { Leitfähig- } \\
\text { keit }\end{array}$ & Typ: 1 & 2 & 3 & $4+5$ & $1+2+3$ & $4+5$ \\
\hline$[\mu \mathrm{A} / \mathrm{F}]$ & 328 & 169 & 289 & 153 & 262 & 153 \\
{$[\mu \mathrm{A} / \mathrm{V}]$} & 302 & 118 & 264 & 135 & 228 & 135 \\
\hline
\end{tabular}

Tab. 2. Mittlere Leitfähigkeit, ausgedrückt in $\mu \mathrm{A} / \mathrm{F}$ und $\mu \mathrm{A} / \mathrm{V}$; a) der einzelnen Typen und b) Typ $1+2+3$ und $4+5$ zusammengefaßt.

Bei der Präparierung der Kristalle wurde darauf geachtet, wie der Verlauf der $c$-Achse zur Feldrichtung liegt. Mit wenigen Ausnahmen erfolgte die Präparierung so, daß die Wachstumsrichtung der Kristalle in Feldrichtung verläuft. Einige wenige Kristalle vom Typ 2 wurden jedoch auch so präpariert, daß die $c$-Achse parallel zum Verlauf der elektrischen Feldlinien lag. Die Kenntnis der Lage der $c$-Achsen war vor allen Dingen auch wichtig für die Frage des Gleichrichtereffektes, den wir bei allen Kristallen bis auf ganz wenige Ausnahmen zunächst in Luft beobachteten. Auch im Vakuum (ca. $10^{-2} \mathrm{~mm}$ $\mathrm{Hg}$ ) war noch in den meisten Fällen ein - wenn auch sehr viel kleinerer - Unterschied der Photo-

Abb. 2 auf Tafel S. 436 a. 
ströme in beiden Richtungen zu beobachten. Im Hochvakuum dagegen verschwand nach ca. 20 Min. an etwa $70 \%$ aller Kristalle der Unterschied vollkommen.

\section{Diskussion}

Sicher könnten noch viele ergänzende Versuche dazu beitragen, um für einzelne Beobachtungen eine genaue Erklärung zu finden. Es kam uns aber, wie schon an anderer Stelle betont, im Rahmen der Aufgabenstellung unseres Institutes darauf an, brauchbare Meßzellen zu schaffen, wobei die Auswahl der Kristalle zunächst nach der Größe der photoelektrischen Leitfähigkeit erfolgen sollte, wenn auch für bestimmte Zwecke (Dosimetrie) die Konstanz der Leitfähigkeitseigenschaften eines Kristalls eine mindestens ebenso große Rolle spielt wie diese. - Die Untersuchung einer größeren Anzahl von Kristallen, wie wir sie vorgenommen haben, sollte uns die Möglichkeit geben, bestimmte Gruppen von Kristallen schon auf Grund ihrer äußeren Erscheinungsform als für weniger geeignet auszuschließen.

Vergleicht man zunächst die Leitfähigkeit der einzelnen Typen im Hochvakuum, so kommt man nach Tab. 2 zu dem Schluß, daß die Leitfähigkeit der Zwillinge im Mittel schlechter ist als die der Einkristalle. Am besten leiten die glasklaren homogenen Einkristalle vom Typ 1. Es fällt auf, daß die Einkristalle vom Typ 2 - Riefelung rechtwinkelig zur Wachstumsrichtung - ein schlechteres Leitvermögen aufweisen als die vom Typ 1 und 3 . Dies kann möglicherweise mit der Lage der $c$-Achse zur Feldrichtung zusammenhängen, da allein bei den Kristallen vom Typ 2 diese immer senkrecht zu den Feldlinien verläuft. Leitfähigkeitsmessungen an drei Kristallen vom Typ 2, bei welchen die Elektroden so aufgedampft waren, daß $c$-Achse und Feldrichtung parallel miteinander verlaufen, zeigten tatsächlich im Mittel eine um 25\% größere Leitfähigkeit.

Es liegt auch nahe, den in Luft und Vakuum $\left(10^{-2} \mathrm{~mm} \mathrm{Hg}\right)$ bei allen Kristallen beobachteten Gleichrichtereffekt auf Grund der Kristallstruktur zu klären und hierfür die polare $c$-Achse verantwortlich zu machen. Ein Hinweis dafür, daß der Gleichrichtereffekt reell ist und nicht auf Kontaktfehlern beruht, ist einmal die Tatsache, daß der Gleichrichtereffekt im Hochvakum verschwindet und daß der Photostrom, wie an $30 \mathrm{Zwillingskristal-}$ len beobachtet, immer dann größer war, wenn der Pluspol der Saugspannung in der Winkelöffnung lag.

Da im Hochvakuum die Richtungsabhängigkeit der Leitfähigkeit verschwindet und die photoelektrischen Ströme bis auf einen konstanten Endwert sinken, kann als Ursache für den Gleichrichtereffekt die in Luft zusätzliche äußere Leitfähigkeit angenommen werden. Diese entsteht, wie auch schon von anderen Autoren beobachtet, durch äußere atmosphärische Einflüsse. Vermutlich werden Wassermoleküle infolge der polaren $c$-Achse polar orientiert von der Kristalloberfläche adsorbiert. Man müßte demnach bei im Hochvakuum befindlichen Kristallen den Gleichrichtereffekt sofort zurückerhalten, wenn man Wasserdampf zusetzt. In einer trockenen Stickstoffatmosphäre dürfte hingegen der Gleichrichtereffekt nicht auftreten. Orientierende Experimente bestätigten dies: Untersuchungen an fünf Kristallen zeigten, daß bei Zugabe von geringen Mengen Wasserdampf der Gleichrichtereffekt sofort auftrat, hingegen bei Aufbewahrung dieser Kristalle in Stickstoffatmosphäre selbst nach 4 Stunden keine Änderung der Leitfähigkeit zu beobachten war. Wenn eine polare Adsorption durch die Polarität der $c$-Achse hervorgerufen wird, so müßte auch die Größe des Gleichrichtereffektes von der Lage der polaren $c$ Achse zur Feldrichtung abhängen. Vergleicht man hierzu die an Luft gemessenen Photoströme in beiden Richtungen, so zeigt sich, daß die Größe des Gleichrichtereffektes - ausgedrückt durch das Verhältnis der Photoströme in beiden Richtungen bei Einkristallen vom Typ 2 (c-Achse senkrecht zur Feldrichtung) dreimal so klein ist als an Einkristallen vom Typ 1 und 3 (c-Achse, bis auf wenige Ausnahmen des Typs 1, immer winkelig zur Feldrichtung). 